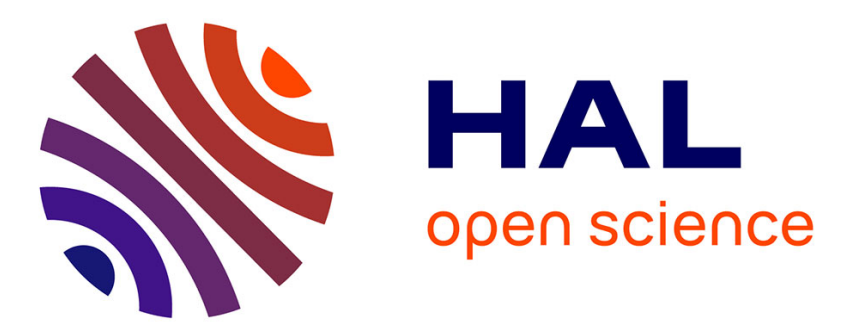

\title{
On the Eigenfrequencies of an Ordered System of Nano-objects
}

Victor A. Eremeyev, Holm Altenbach

\section{To cite this version:}

Victor A. Eremeyev, Holm Altenbach. On the Eigenfrequencies of an Ordered System of Nano-objects. 2009, pp.123-132. hal-00824013

\section{HAL Id: hal-00824013 \\ https://hal.science/hal-00824013}

Submitted on 20 May 2013

HAL is a multi-disciplinary open access archive for the deposit and dissemination of scientific research documents, whether they are published or not. The documents may come from teaching and research institutions in France or abroad, or from public or private research centers.
L'archive ouverte pluridisciplinaire HAL, est destinée au dépôt et à la diffusion de documents scientifiques de niveau recherche, publiés ou non, émanant des établissements d'enseignement et de recherche français ou étrangers, des laboratoires publics ou privés. 


\title{
On the Eigenfrequencies of an Ordered System of Nanoobjects
}

Victor A. Eremeyev and Holm Altenbach

\begin{abstract}
A method is discussed to determine the eigenfrequencies of nanostructures (nanotubes, nanospheres, and nanocrystals) by measuring the eigenfrequencies of a 'large system' that consists of an array of vertically oriented similar nanotubes or nanocrystals equidistantly grown on a substrate. It is shown that the eigenfrequencies of a single nanoobject can be derived from the eigenfrequency spectra of the large (array-substrate) system and of the substrate. With other words, using experimental data for large systems one can determine the eigenfrequencies of a single nanoobject. The method can be also applied to systems of nanotubes grown in parallel to the substrate and to the systems of micro- and nanospheres. The modeling of nanocomposite plates using the direct approach to the shell theory is discussed. The effective stiffness tensors are considered. As an example, the eigenfrequencies of an array of $\mathrm{ZnO}$ micro- or nanocrystals and GaAs multiwalled nanotubes on a sapphire substrate are calculated.
\end{abstract}

\section{Introduction}

The experimental determination of the mechanical characteristics of nanoobjects is today a challenging problem (see, among others, $[6,7,14,16,17,20])$. One of the most efficient methods to determine elastic moduli in macromechanics is the measurement of the eigenfrequencies of an object. However, attempts to apply this approach to nanoobjects sometimes demonstrate difficulties. There are at least two problems lying at the interfaces between mechanics and experimental physics. The

Victor A. Eremeyev

South Scientific Center of RASci South Federal University, 8a Milchakova st., Rostov on Don 344090, Russia; e-mail: eremeyev.victor@gmail.com

Holm Altenbach

Lehrstuhl für Technische Mechanik, Zentrum für Ingenieurwissenschaften, Martin-LutherUniversität Halle-Wittenberg, D-06099 Halle (Saale), Germany 


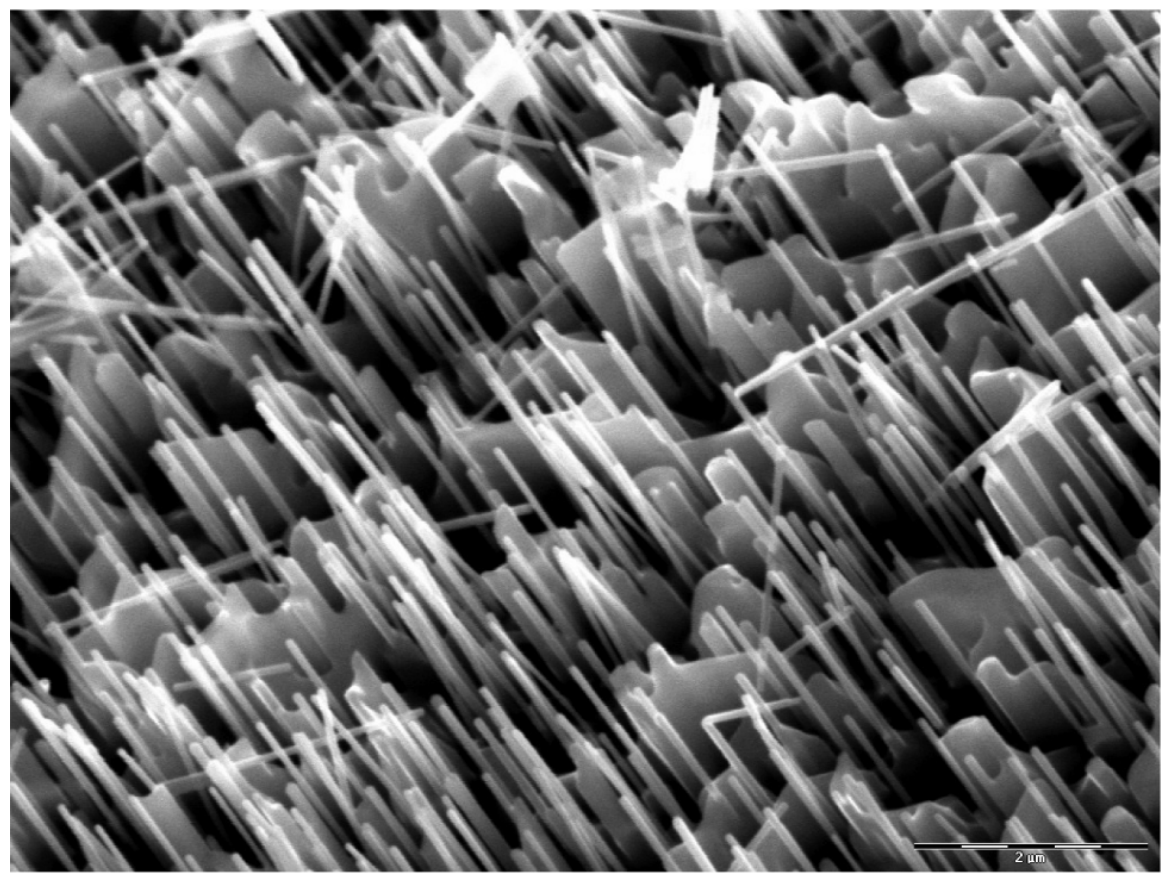

Fig. 1 Example of nanoobject array: $\mathrm{ZnO}$ nanocrystals on a substrate (photos by courtesy of Konstantin Dvadnenko, South Scientific Center of RASci).

first one is the determination of the elastic moduli of nanoobjects when there is a possibility to measure the frequencies of the microsubstrate-nanoarray system and determine the elastic characteristics of the substrate (for example, from the eigenfrequencies of the free substrate). The second problem is how to extract the eigenfrequencies of nanoobjects from the eigenfrequency spectrum of the substrate-array system. The success in solving both problems directly depends on the experimental conditions, especially, on the way how nanoobjects are grown on the substrate and how the substrate with nanoobjects is fixed in the measuring device; as well as on the geometries, weights, and the elastic properties of the nanoobjects and the substrate. Thus, from the mechanical point of view, one should not only discuss the measured data, but also elaborate a suitable design of the experiments.

Here we discuss the method of determining the eigenfrequencies of nanostructures (nanotubes and nanocrystals) from the measured eigenfrequencies of a large system comprising a highly ordered array of identical nanospheres, nanotubes or nanocrystals grown on a substrate proposed in [10-13]. The geometry of the considered nanostructures is presented in Figure 2. An example of such large system, the array of nanocrystals of zinc oxide, is presented in Figure 1. $\mathrm{ZnO}$ nanocrystals are of considerable interest for nanomechanics and nanophotonics and can be fabricated by different techniques. The array of GaAs, GeSi nanotubes growing horizontally on a substrate may be obtained by technology described in $[15,21]$. The interest to 


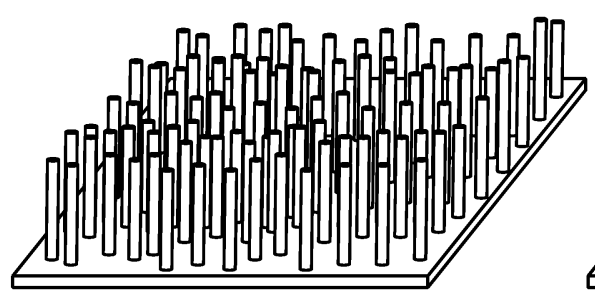

c

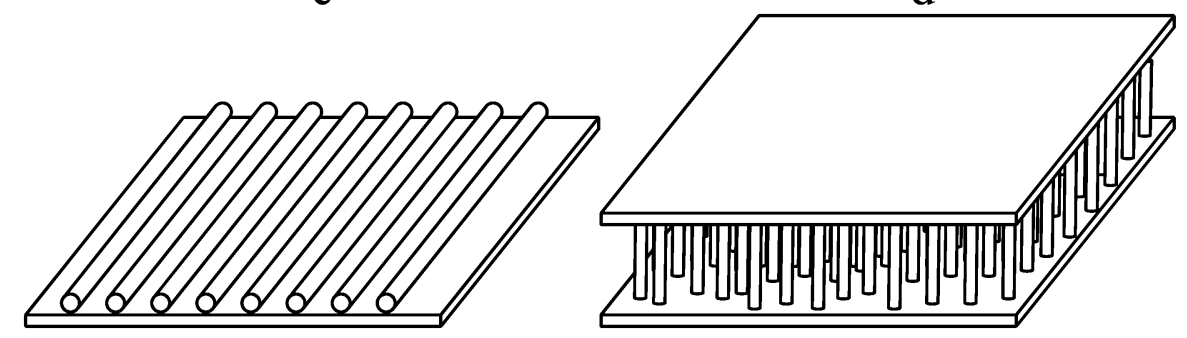

Fig. 2 Nanostructures: (a) an array of nanotubes or nanocrystals on substrate; (b) an array of spherical micro- or nanoparticles; (c) an array of nanotubes lying on a substrate; (d) nanocomposite plate.

the arrays of hollow sphere-like nanoparticles is motivated by possible applications to modeling of fullerenes, dendrimers, micelles, vesicles, and liposomes. Some of them are widely used in modern medicine and farmacology, for example, in order to deliver encapsulated drugs to target organs in the organism.

Using the asymptotic solutions based on the shell theory it is shown that from the spectrum of the large system (nanoobjects-substrate), the eigenfrequencies of an individual nanoobject can be derived. That means, the eigenfrequencies of a single nanoobject can be determined from experimental data obtained for the large system. The direct FEM calculation results also indicate the possibility of extracting the spectrum of nanoobjects from the spectrum of the large system.

The oriented arrays of nanoobjects can be used for assembling of nanocomposite thin-walled structures, for example, plates (see Figure 2d). In the last section of this paper we discuss the methods of identifications of elastic properties of a nanocomposite plate using the approach [1-3,23].

\section{Governing Equations of the Plate and Shell Theory}

We apply the theory of plates and shells formulated earlier in [1-5, 22, 23]. From the direct approach point of view a plate or a shell is modeled as a material surface each particle of which has five degrees of freedom (three displacements and two 
rotations, the rotation about the normal to plate is not considered as a kinematically independent variable). Note that the application of the direct approach to the thinwalled nanostructures has advantages because it is free from the consideration of the nanostructure on the base of the three-dimensional continuum mechanics.

The equations of motion are formulated as the Euler's laws of dynamics:

$$
\nabla \cdot \mathbf{T}+\mathbf{q}=\rho \ddot{\mathbf{u}}+\rho \boldsymbol{\Theta}_{1} \cdot \ddot{\boldsymbol{\varphi}}, \quad \nabla \cdot \mathbf{M}+\mathbf{T}_{\times}+\mathbf{m}=\rho \boldsymbol{\Theta}_{1}^{T} \cdot \ddot{\mathbf{u}}+\rho \boldsymbol{\Theta}_{2} \cdot \ddot{\boldsymbol{\varphi}} .
$$

Here $\mathbf{T}, \mathbf{M}$ are the tensors of forces and moments, $\mathbf{q}, \mathbf{m}$ are the vectors of surface loads (forces and moments), $\mathbf{T}_{\times}$is the vector invariant of the force tensor, $\nabla$ is the nabla (Hamilton) operator, $\mathbf{u}, \boldsymbol{\varphi}$ are the vectors of displacements and rotations, $\boldsymbol{\Theta}_{1}$, $\boldsymbol{\Theta}_{2}$ are the first and the second tensor of inertia, $\rho$ is the density (effective property of the deformable surface), $(\ldots)^{T}$ denotes transposed and $(\cdot)^{\bullet}$ the time derivative. The geometrical equations are given as

$$
\boldsymbol{\mu}=(\nabla \mathbf{u} \cdot \mathbf{a})^{\mathrm{sym}}, \quad \boldsymbol{\gamma}=\nabla \mathbf{u} \cdot \mathbf{n}+\mathbf{c} \cdot \boldsymbol{\varphi}, \quad \boldsymbol{\kappa}=\nabla \boldsymbol{\varphi} .
$$

$\mathbf{a}$ is the first metric tensor (plane tensor), $\mathbf{n}$ is the unit outer normal vector at the surface, $\mathbf{c}$ is the discriminant tensor $(\mathbf{c}=-\mathbf{a} \times \mathbf{n}), \boldsymbol{\mu}, \boldsymbol{\gamma}$ and $\boldsymbol{\kappa}$ are the strain tensors (the tensor of in-plane strains, the vector of transverse shear strains and the tensor of the out-of-plane strains), while $\mathbf{t}^{\text {sym }}$ denotes the symmetric part.

The boundary conditions are given by

$$
\boldsymbol{v} \cdot \mathbf{T}=\mathbf{f}, \quad \boldsymbol{v} \cdot \mathbf{M}=\mathbf{l}(\mathbf{l} \cdot \mathbf{n}=0) \quad \text { or } \quad \mathbf{u}=\mathbf{u}^{0}, \quad \boldsymbol{\varphi}=\varphi^{0} \text { along } S .
$$

Here $\mathbf{f}$ and $\mathbf{l}$ are external force and moment vectors acting along the boundary of the plate $S$, while $\mathbf{u}^{0}$ and $\varphi^{0}$ are given functions describing the displacements and rotations of the plate boundary, respectively. $\boldsymbol{v}$ is the unit outer normal vector to the boundary $S(\boldsymbol{v} \cdot \mathbf{n}=0)$. The relations (3) are the static and the kinematic boundary conditions. Other types of boundary conditions are also possible. The constitutive equations are given by the following relations:

Strain energy of the deformable surface $W$ :

$W(\boldsymbol{\mu}, \boldsymbol{\gamma}, \boldsymbol{\kappa})=\frac{1}{2} \boldsymbol{\mu} \cdot \mathbf{A} \cdot \boldsymbol{\mu}+\boldsymbol{\mu} \cdot \mathbf{B} \cdot \boldsymbol{\kappa}+\frac{1}{2} \kappa \cdot \mathbf{C} \cdot \boldsymbol{\kappa}+\frac{1}{2} \boldsymbol{\gamma} \cdot \boldsymbol{\Gamma} \cdot \boldsymbol{\gamma}+\boldsymbol{\gamma} \cdot\left(\boldsymbol{\Gamma}_{1} \cdot \boldsymbol{\mu}+\boldsymbol{\Gamma}_{2} \cdot \boldsymbol{\kappa}\right)$.

$\mathbf{A}, \mathbf{B}, \mathbf{C}$ are fourth rank tensors, $\boldsymbol{\Gamma}_{1}, \boldsymbol{\Gamma}_{2}$ are third rank tensors, $\boldsymbol{\Gamma}$ is a second rank tensor of the effective stiffness properties. They depend on the material properties and the cross-section geometry. In the general case the tensors contain 36 different values - a reduction is possible assuming some symmetries.

Constitutive equations

- In-plane forces

$$
\mathbf{T} \cdot \mathbf{a}=\frac{\partial W}{\partial \boldsymbol{\mu}}=\mathbf{A} \cdot \boldsymbol{\mu}+\mathbf{B} \cdot \boldsymbol{\kappa}+\boldsymbol{\gamma} \cdot \boldsymbol{\Gamma}_{1}
$$


- Transverse forces

$$
\mathbf{T} \cdot \mathbf{n}=\frac{\partial W}{\partial \boldsymbol{\gamma}}=\boldsymbol{\Gamma} \cdot \boldsymbol{\gamma}+\boldsymbol{\Gamma}_{1} \cdot \boldsymbol{\mu}+\boldsymbol{\Gamma}_{2} \cdot \boldsymbol{\kappa}
$$

- Moments

$$
\mathbf{M}^{T}=\frac{\partial W}{\partial \boldsymbol{\kappa}}=\boldsymbol{\mu} \cdot \mathbf{B}+\mathbf{C} \cdot \boldsymbol{\kappa}+\boldsymbol{\gamma} \cdot \boldsymbol{\Gamma}_{2} .
$$

\section{On the Modeling of Natural Oscillations of an Array of Nanoobjects}

For the analytical investigation of such complex structures as presented in Figure 1 we have to make some assumptions on the stress state and the possible deformations of the shell-like structure. Here we have been used the models of the beams, of spherical and cylindrical shells for the nanocrystals, the nanotubes and the nanospheres as well as the plate equations for the substrate. To avoid the awkward calculations we present here the final result: for all of three cases we may choose the geometry of the large system consisting of an array of nanoobjects in such a way that one may find the first eigenfrequencies of the nanoobject from its spectrum. The accuracy of determination of the first eigenfrequency for the vertically oriented $\mathrm{ZnO}$ crystals is about $5 \%$, while for the GaAs nanotubes lying horizontally the accuracy is about $10-15 \%$ (for details, see [10-13]).

Note that the behavior of the system with horizontally located nanotubes differs from the behavior of a similar system with vertically located nanotubes [10], as well as from the behavior a system of nanospheres [11], because the horizontally attached nanotubes change the effective stiffness of the plate and the plate with the horizontal nanotubes is anisotropic and inhomogeneous with respect to the effective properties.

For an arbitrary stress-strain state we investigate the system consisting of a certain number of nanoobjects on the substrate in the framework of the threedimensional theory by means of the FEM. Since the materials under consideration ( $\mathrm{ZnO}, \mathrm{GaAs}$, InAs, GeSi, etc.) have piezoelectric properties, these systems as a whole are a composite piezoelectric solids. Then for modeling of GaAs nanotubes and $\mathrm{ZnO}$ nanocrystals we used the constitutive equations of the theory of anisotropic electroelasticity $[18,19]$.

As an example of FEM modeling let us consider the array of horizontal GaAs nanotubes (Figure 2c). For the modal analysis of the corresponding boundary value problems we have used FE code ANSYS. Various numerical experiments are performed for different mesh, different types of finite elements, different numbers of nanotubes (from one to ten), different system geometries (the ratio of the thicknesses of the substrate and the nanofilm, the ratio of the nanotube radius to the substrate length, etc.), and various substrate bonding conditions. We consider the same materials of the substrate and the nanotubes used in $[15,21]$. The properties of these 

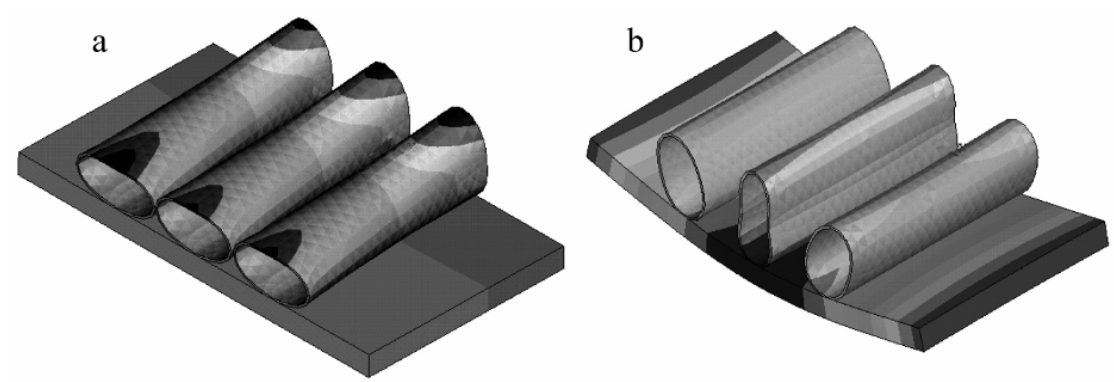

Fig. 3 Sapphire substrate with three GaAs nanotubes: (a) natural mode localized in nanotubes; (b) natural mode corresponding to the first bending mode of the substrate.

materials are taken from [8], and the geometrical parameters are taken from [15,21]. The calculations show that for any bonding of the substrate, it is possible to choose the problem parameters in such a way that the natural frequencies of the nanotubes and the substrate can be extracted from the general spectrum of the large system. The numerical estimates confirm the results of the above theoretical analysis.

The calculation results for the free sapphire substrate with three GaAs nanotubes are shown in Figure 3. Figure 3a corresponds to the natural vibrations localized in the nanotubes. Figure $3 \mathrm{~b}$ shows the natural vibrations at the frequency corresponding to the first bending mode of the substrate vibrations.

The eigenmodes for the nanospheres are presented in Figure 4. It is easy to see that we have approximately the same situation as shown in Figure 3. The spectrum of eigenmodes consists of modes which are localized in a sphere and the modes of the substrate. Let us note that the separation of the eigenmodes is fulfilled only for the first part of the spectrum when the localized motion of the array is possible. The high-oscillating modes do not correspond to any modes of a single nanoobject or the substrate. The high-oscillating modes depend on the interaction of the nanoarray and the substrate. Note that the vibration interaction between the substrate and nanotubes is stronger than that for a case of a vertical array of nanocrystals or nanospheres.

The eigenfrequencies for different nanoarrays are summarized in Figure 5. Here the distribution of the natural frequencies $\omega$ over their ordinal numbers $n$ is depicted. In Figure 5a the results of the modal analysis of the large system consisting of 8 $\mathrm{ZnO}$ nano-sized crystals grown on a sapphire substrate are presented, see [10]. The eigenfrequencies of three GaAs nanotubes attached to the sapphire substrate are given in Figure 5b, while in Figure 5c the eigenfrequencies of 33 hollow spheres made of a polymer are presented. The graphs show the presence of a plateau in the distributions. The length of the plateau depends on the number of nanoobjects in the array. The plateau corresponds to the eigenfrequency of one nanoobject. A big number of approximately equal eigenfrequencies gives us additional chance to find it by using of experimental measurements. On the other hand if one extends the graphs to the higher n-values the next plateaus will be disappeared. For example, the second plateau for the array of nanotubes is not strictly expressed. But for the first frequencies, the numerical coincidence of the eigenfrequencies of the large system 


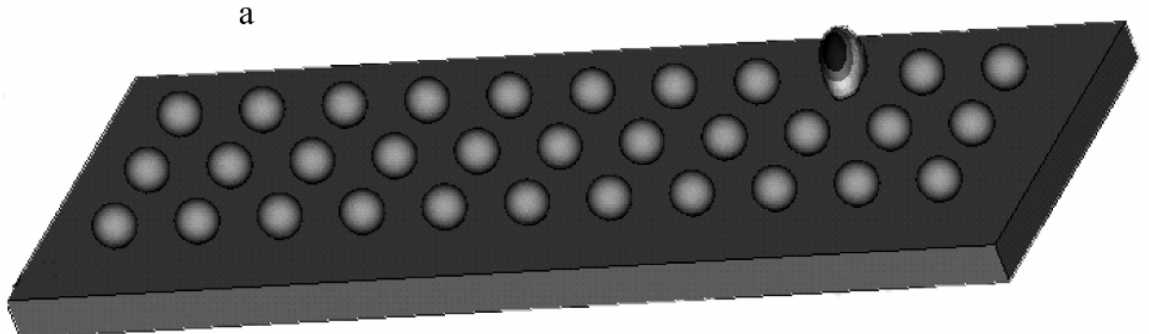

b

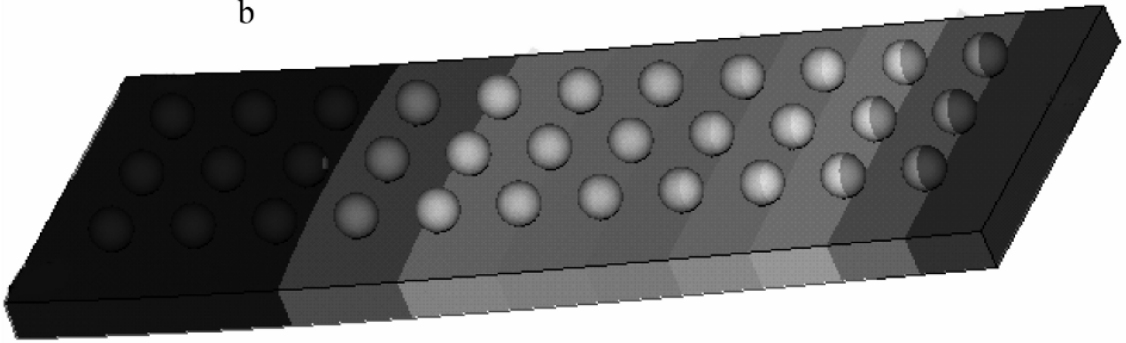

Fig. 4 Eigenmodes of a substrate with nanospheres: (a) natural mode localized in one nanosphere; (b) natural mode corresponding to the first bending mode of the substrate.

with the respective partial eigenfrequencies of the substrate and single nanotube is satisfactory.

From an engineering point of view, let us mention that the main restriction on application of the described method is the frequency range of measuring instruments. If the eigenfrequencies of the nanoobjects are too high, such frequencies cannot be registered. On the other hand, the method is the most effective and accurate method if the first eigenfrequencies of the nanoobjects are comparable with the first eigenfrequencies of the substrate. Thus, the governing factor in using this method is a good choice of proportions between both the geometric and the physical characteristics of the nanoobjects and the substrate.

\section{Effective Properties of Nanocomposite Plates}

In $[1-3,23]$ a new approach to the determination of the effective stiffness tensors was proposed. The idea of this approach is based on the comparison of the exact solutions of the three-dimensional elasticity and the corresponding solutions of Equations (1)-(6) for several test problems of the elastostatics such as tension and bending, plane shear, and torsion. The method was applied to isotropic plates as well as to orthotropic and transversally isotropic material behavior. Now it is well known that the nanocomposites demonstrate mechanical properties which may be quite different from its macro-analogues, see e.g. [7, 17]. One of the reasons of 
$\omega 10^{-1} \mathrm{GHz}$

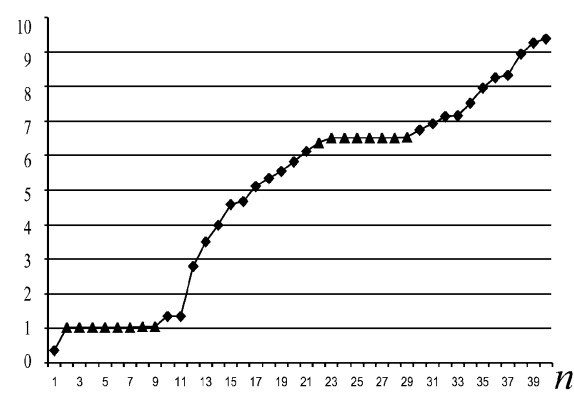

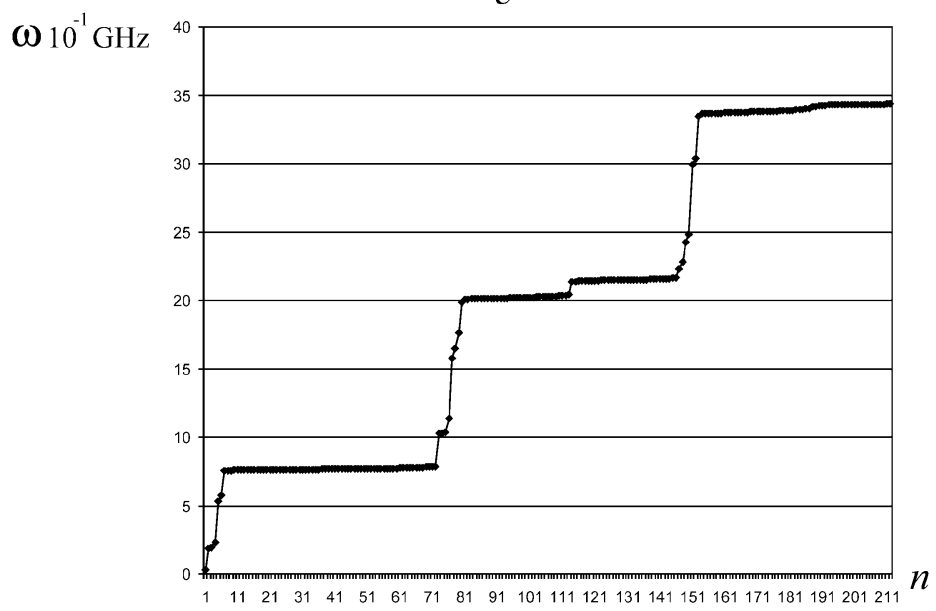

Fig. 5 Distribution of the natural frequencies of the large system vs. their number $n$ : (a) for $\mathrm{ZnO}$ nanocrystals; (b) for GaAs nanotubes lying horizontally (the squares are the natural frequencies corresponding to the vibrations of the substrate); (c) for spherical nano-membranes.

such difference is the surface tension, because these materials have a fractal-like surface. The influence of the surface tension on the elastic moduli was investigated by several authors, see for example [9]. From this point of view they may consider the nanocomposite based on an oriented array of nanocrystals or nanotubes as a transversally isotropic material. We propose to extend the procedure $[1,2,23]$ to the case of such materials. Finally, one obtains the components of the effective stiffness tensors which depend not only on the bulk elastic properties and the thickness of the plate but also on the surface tension. 
A direct approach method in the theory of shells for nano-sized thin-walled structures is introduced. The basic items of the theory are related to:

- the formulation of all balances for a deformable directed surface (a priori twodimensional equations), and

- the specific constitutive equations.

The direct approach has an advantage to the modeling of nanostructures because it is not necessary to consider nanostructures as three-dimensional solids, while many of them are not existed in the bulk phase or the properties of the bulk phase are quite different from the properties of the nanoobjects. On the other hand the determination of the elastic stiffness tensors is essential for the applicability of the theory. The effective stiffness may be obtained by consideration of experimental data on eigenfrequencies. Here we discussed a possible method of determination of the eigenfrequencies of single nanoobjects by investigation the spectrum of the large system consisting of an array of identically nanoobjects grown on a substrate.

The theoretical and numerical analysis performed for nanospheres, nanocrystals and nanotubes, shows that it is possible to determine the eigenfrequencies of microand nanoobjects in experiments using the measurement of eigenfrequencies of the substrate with an array of such nanodimensional objects bonded to the surface. The results of numerical calculations of the eigenfrequencies and the eigenmodes are presented. The identification procedure of the effective stiffness of nanocomposite plates is discussed.

Acknowledgements The research work was partially supported by the Martin-Luther-University Halle-Wittenberg and the program of development of the South Federal University.

\section{References}

1. Altenbach, H.: An alternative determination of transverse shear stiffnesses for sandwich and laminated plates. Int. J. Solids Struct. 37, 3503-3520 (2000).

2. Altenbach, H.: On the determination of transverse shear stiffnesses of orthotropic plates. ZAMP 51, 629-649 (2000).

3. Altenbach, H., Eremeyev, V.A.: Direct approach based analysis of plates composed of functionally graded materials. Arch. Appl. Mech. 37, 775-794 (2008).

4. Altenbach, H., Zhilin, P.: A general theory of elastic simple shells. Usp. Mekh. 11, 107-114 (1988) [in Russian].

5. Altenbach, H., Zhilin, P.: The theory of simple elastic shells. In: Kienzler, R., Altenbach, H., Ott, I. (Eds.), Critical Review of the Theories of Plates and Shells and New Applications, Lect. Notes Appl. Comp. Mech. Vol. 16, pp. 1-12. Springer, Berlin (2004).

6. Balzani, V., Credi, A., Venturi, M.: Molecular Devices and Machines. A Journey into the Nano World. Wiley, Weinheim (2003).

7. Bhushan, B. (Ed.): Springer Handbook of Nanotechnology. Springer, Berlin (2004).

8. Dargys, A., Kundrotas, J.: Handbook of Physical Properties of Ge, Si, GaAs, and InP, Sci. and Encyclopedia Publ., Vilnius (1994). 
9. Duan, H.L., Wang, J., Karihaloo, B.L., Huang, Z.P.: Nanoporous materials can be made stiffer than non-porous counterparts by surface modification. Acta Mater. 54, 2983-2990 (2006).

10. Eremeyev, V.A., Ivanova, E.A., Morozov, N.F., Soloviev, A.N.: Method of determining the eigenfrequencies of an ordered system of nanoobjects. Techn. Phys. 52, 1-6 (2006).

11. Eremeyev, V.A., Ivanova, E.A., Morozov, N.F., Strochkov, S.E.: The spectrum of natural oscillations of an array of micro- or nanospheres on an elastic substrate. Dokl. Phys. 52, 699-702 (2007).

12. Eremeyev, V.A., Ivanova, E.A., Morozov, N.F., Strochkov, S.E.: Natural vibrations of nanotubes. Dokl. Phys. 52, 431-435 (2007).

13. Eremeyev, V.A., Ivanova, E.A., Morozov, N.F., Strochkov, S.E.: Natural vibrations in a system of nanotubes. J. Appl. Mech. Techn. Phys. 49, 291-300 (2008).

14. Goddard, W.A., Brenner, D.W., Lyshevski, S. E., Iafrate, G.J. (Eds.): Handbook of Nanoscience, Engineering, and Technology. CRC Press, Boca Raton (2003).

15. Golod, S.V., Prinz, V.Ya., Mashanov, V.I., Gutakovski, A.K.: Fabrication of conducting GeSi/Si micro- and nanotubes and helical microcoils. Semiconductor Sci. Technol. 16, 181185 (2001).

16. Harik, V.M., Salas, M.D. (Eds.): Trends in Nanoscale Mechanics. Analysis of Nanostructured Materials and Multi-Scale Modeling. Kluwer, Dordrecht (2003).

17. Harris, P.: Carbon Nanotubes and Related Structures: New Materials for the Twenty-First Century. Cambridge University Press, Cambridge (2001).

18. Maugin, G.A.: Continuum Mechanics of Electromagnetic Solids. North-Holland, Amsterdam (1988).

19. Nowacki W.: Electromagnetic Effects in Deformable Solids. PWN, Warsaw (1983) [in Polish].

20. Poole, C., Jr., Owens, F., Jr.: Introduction to Nanotechnology. Wiley, Hoboken NJ (2003).

21. Prinz, V.Ya.: A new concept in fabricating building blocks for nanoelectronics and nanomechanics devices. Microelectron. Engrg. 69, 466-475 (2003).

22. Zhilin, P.A.: Mechanics of deformable directed surfaces. Int. J. Solids Struct. 12, 635-648 (1976).

23. Zhilin, P.A.: Applied Mechanics. Foundations of the Theory of Shells. St. Petersburg State Polytechnical University, St. Petersburg (2006) [in Russian]. 